\title{
从对电视剧《施琅大将军》的争论看 中国文化民族主义复兴的困境
}

高墨波

With the rise of China's status on international stage as a result of its economic development there is an increasingly audible voice of Chinese cultural nationalism. More and more educators and intellectuals are calling for the revival of Chinese traditional culture values. However, the very issues of who are the Chinese and what are traditional Chinese cultural values present a huge dilemma. The debates on a recent a TV program "General Shi Lang" consist a good case to illustrate this dilemma. Shi Lang was a general of the last days of the Ming Dynasty, but had surrendered to the Manchu Qing dynasty invaders and fought for the Machus take over Taiwan from a Chinese ruler. The debates show that the dilemma has its cause in at least three issues. First, it is not at all obvious who should be categorized as Chinese. Secondly, it is not at all obvious what Chinese cultural values are. And finally it is the issue of evaluation of traditional values against the universal claim of the values of liberal democracy.

\section{引论}

中国人 ${ }^{1}$ 的民族危机感是在西洋和东洋帝国主义和殖民主义的炮火轰倒了中国 的前门、后门和侧门以后才迸发出来的。在此之前, 中国并没有一个民族国家 的概念。中国人一一应该说是有儒学知识的一一国人有一个天下的概念和一 个中华文化的概念。在这两个概念里，凡不属于中华文化圈子里的都是夷。

虽然统治这个文化圈子的王权时常遭北方游牧族的侵犯, “我” 与 “他” 的区 分不是以国家疆界而定的, 比喻归顺了的进贡国也可能是 “我”。也不是以种 族来区分的, 不论哪个种族拿到了王权的正统权就是中华文化里的国人。因为

\footnotetext{
1 什么人是中国人是个要讨论的复杂问题, 参看高默波, 'Chinese What Chinese: The Politics of Authenticity and Ethnic Identity’, in Lee Guan Kin, ed., National Boundaries and Cultural Configurations, Centre for Chinese Language and Culture and Global Publishing Co. Pte. Ltd, 2006. 2 这种夷原本没有歧视的意思。参见伍国: 《略说孔夫子的民族国家观念》，世纪中国网 http://www.cc.org.cn/newcc/browwenzhang.php?articleid=7084，2006 年 7 月 11 日查阅。
} 
如此, 蒙古人统治的元朝和满人统治的清朝都是历史的中国和中国的历史。也 因为如此, 中国这个实体的疆界有时大有时小。同样的原因, 很长一段时间, 台湾属不属于中国都无所谓。以后割让给日本在当时也没有当作大不了的事。 这个历史给我们遗留下一个什么是民族国家的难题。这个难题是中国文化民族 主义复兴的第一个原因。

这种文化历史路径使中国文人以文化价值来判断先进与落后, 现代与传统, 文 明和非文明。我们的邻居日本就不同。他们并没有民主的传统, 也没有个人主 义自由的文化价值。 ${ }^{3}$ 但是他们并没有因此而疼骂祖宗三代。他们先是借用中 国文化，后是借用西方文化，并把借用的东西改成自己的传统，以此为自豪。 中国的民族主义者从孙中山的反满复兴到五四运动的打倒孔家店, 从胡适的全 盘西化到鲁迅把中国礼教谴责成吃人的制度, ${ }^{4}$ 无不是在文化上论是非。而当 代中国文革后的告别革命以及赵紫阳支持的《河歾》， ${ }^{5}$ 也是在算文化的帐。 把中国革命的起因说成是救亡压倒启蒙无非是说, 中国革命的失败是必然的, 因为中国文化的启蒙还不够彻底。比如 “刘小枫说, 五四时代的一个最大错 误，就是没有把基督教搬进来。所以他希望基督教来救中国”。 ${ }^{6}$ 中国精英民 族主义者 主流 $^{7}$ 一直在制造这种中国古代和现代民族文化虚无主义 ${ }^{8}$ 的真理。 很多人并没有觉察到这民族主义真理话语的自相矛盾: 为了中国民族的兴起中 国人必须彻底批判和抛弃中国的民族文化!

这个自相矛盾之所以没有被觉察, 是因为这些先进的中国人认为, 西方文化价 值是普世的, 永恒的和超国界的。虽然后现代主义和后殖民主义思想和方法对 中国文化虚无主义进行了努力不小的反击 ${ }^{9}$, 虽然中国也有西方主义的现象, ${ }^{10}$ 西方文化价值是普世的、永恒的和超国界的思潮现在在中国的知识界还是占统 治地位。所不同的是, 很多持这种观点的人不再称自己是中国民族主义者。而 西方强大的物质财富和次序井然的民主自由公民生活使这真理的真理性一目了 然和无解可击。以西方文化为标准把中国民族文化判断为反现代文明和反现代 的。这是必然使中国文化民族主义复兴陷入困境的第二个原因。

\footnotetext{
${ }^{3}$ Mobo Gao, 'Democracy, What Democracy? China's Road to Modernisation', China Report (New Delhi), Vol. 28, No. 1 (Jan.-March) 1992, pp. 13-26.

4 见鲁迅《狂人日记》、《阿Q正传》和《祥林嫂》等。

$5^{5}$ 李泽厚: 《告别革命: 回望二十世纪中国》, 香港: 天的图书有限公司, 2004 年第 5 版。

6 黄锫坚: 《李泽厚: 马克思我的思想基点之一》, 白鹿书院,

http://www.bailushuyuan.com/html/xwwh/2005126150211.htm，2006 年 7 月 10 日引用。

7 这里主要是指非政府的知识分子精英民族主义者。关于当代的政府民族主义参看Guo Yingjie, The politics of national identity in post-Tiananmen China: cultural nationalism v. state nationalism, University of Tasmania, $\mathrm{PhD}$ thesis, 2001. Here Guo makes a crucial difference between cultural nationalism and political nationalism in China. 关于四种不同的中国民族主义参看Townsend, James, "Chinese nationalism" in Jon Unger, ed., Chinese Nationalism, Armonk, New York: M. E. Sharpe, 1996.

${ }^{8}$ 这里是指尼采精神的nihilism, 把中国文化贬低为毫无价值的, 与人类文明有害的。跟文化偶 像破坏主义cultural iconoclasm有相通之处。

${ }^{9}$ Yingjie Guo, Cultural Nationalism in Contemporary China: The Search for National Identity under Reform, London and New York: RoutledgeCurzon, 2004.

${ }^{10}$ Xiaomei Chen, Occidentalism- A Theory of Counter-Discourse in Post-Mao China, New York: Oxford University Press, 1995.
} 
由于西方文化价值普世、永恒和超国界的真理是以物质财富为引证的, 东亚国 家和中国国民经济的上升就使中国文化民族主义有复兴的根据和证据。所以不 奇怪的是，二十世纪九十年代之的民族主义也有重新唤起中国传统文化的号 角。这从郭英杰研究过的曾国藩现象可以看得出来。 ${ }^{11}$ 特别是在告别革命以后 中国需要民族认同和民族同一性。从那以后, 儒教的更生和复兴就一浪高过一 浪。比如有王蒙等等参加发起的《甲午宣言》。可不是, 号召读经的蒋庆已经 是风云人物了。如果说对曾国藩高度颂扬的重新评价是标志了中国进一步从汉 人中心主义到现代中国多民族国家意识转变的话， ${ }^{12}$ 眼下网上对《施琅大将 军》》的争论则说明事转变不容易。不容易的原因是, 尽管现代意识的民族国 家可以把满人、蒙古人甚至西藏人和维吾尔族人包括进去叫中国人, 但是中国 文化圈子包括什么人却是个复杂的问题。这就是中国文化民族主义复兴困境的 第三个主要原因。

本文就是要以对《施琅大将军〉这一电视剧的争议来说明以上所说的中国文化 民族主义复兴之困境的三个原因。

\section{施琅、郑成功、台湾和电视剧《施琅大将军》}

施琅 (1621-1696 年) 是明末清初的军人。和许多那个时代的汉人或是在汉人统 治地区的非汉族军人一样, 施琅面临着满人入侵, 明朝灭亡时道德上的两难之 地: 要么是投降入侵之敌, 要么是战死疆场, 甚至全家毁灭。实际上很多人没 有选择的余地和机会。施琅有过两次降清, 第一次是 1646 年, 施琅随同郑芝龙 （郑成功之父）及其叔施福降清。投降后被编入李成栋部的施氏叔侄为清军立 有不少战功。后施琅因故回乡投靠郑成功, 随后施福也因为施氏兄弟的缘故投 入郑成功的部队。施琅后来与郑成功闹翻, 继而郑剥夺了施琅兵权。及至最后 逮捕了施氏父子三人, 并杀掉了施琅的父亲和兄弟。在部下的帮助下逃脱后施 琅第二次降清。郑成功杀施琅之父弟是出于私人恩怨，还是出于反清 “公 心”，至今还是个争议的问题。

事情的关键是，郑成功和施琅都是因攻打台湾而确定了在中国历史上的地位。 在荷兰人占领台湾之后的 1626 年, 西班牙人也登上台湾岛, 占据北部。荷兰人 最终赶走了西班牙人，获取了对整个台湾的控制权。明亡后，郑成功于 1661 年 开始渡海攻打荷兰占领下的台湾。荷军于次年被打败。郑成功于是撤退到被荷 兰占领 38 年之久的台湾。

郑成功去逝后, 长子郑经与郑成功之弟发生争权斗争。清朝借机招抚郑经, 但 郑经坚持割据。1663 年, 清军任用施琅为主帅, 于 1664 年和 1665 年两次攻台 都不胜而退。海峡两岸开始了长达十几年的谈判。1683 年清政府利用郑经病 亡、台湾内部出现分裂再次出兵攻打台湾。郑军大败。当年 8 月 13 日, 施琅率

\footnotetext{
${ }^{11}$ Yingjie Guo, 'Reimagining the Chinese Nation: The Zeng Guofan Phenomenon', Modern China, vol 2 (April), 1999, pp. 142-70.

12 同上。
} 
清军登陆台湾。于是为期 20 余年的台湾割据局面结束了, 台湾与大陆实现了统 一。

由陈明倡议策划、福建电影制片厂电视部根据颜廷瑞的《威震台海》一书改编 的电视剧《施琅大将军》放映之后立即了争议。陈明承认, 策划《施琅大将 军》是为了古为今用。他的目的是想唤起国人对台湾问题的关注，其次是想振 作决策层对解决台湾问题的意志力和决心。13 陈明也承认, 策划《施琅大将 军》也有商业目的。他 “相信这个电视剧肯定能够挣钱, 因为它本身既有很多 的戏剧性因素，又有很多可以 ‘影射” 的东西。譬如说，满清由外来政权渐渐 认同传统, 李登辉由国民党蜕化为台独; 为了解决台湾问题, 康熙开始选用有 才干的汉人出任军事实职”。14 电视剧《施琅大将军》一经播出就立即引发各 方关注和猜测, 随之网络上展开了一场对施珢历史定位的激烈争论。

\section{争论什么?}

争论的第一点是施琅的个人品格。一种观点认为, 两次投降入侵满人的军人是 品格极坏的, 是汉奸。怎么能以英雄的形象出现在电视银幕上? 而另一观点却 认为, 施琅与郑家的恩怨主要是权力斗争的结果, 当年郑成功杀了施琅一家, 故施琅改而投靠满清, 最后更带领清兵打回台湾。施琅收复台湾后, 并没有伤 害郑家后代, 更在康熙面前建议好好利用郑家, 包括派驻出身海盗、有丰富海 上作战经验的郑家在海事通道为朝廷服务。可以说施琅的人格没有可反对之 处。 ${ }^{15}$ 一个观点认为, 施琅不忠于自己的国家。施跟当时的王夫之 (16191692)、顾炎武 (1613-1682)、黄宗羲（1610～1695) 是同一代人。如果施琅帮助 清廷打下台湾证明了他是 “民族英雄” ，那么当时坚持反清复明，或者誓不与 清廷为伍的三大儒, 又将如何评价? 另一观点则认为, 施琅第一次降清不是主 动的, 是跟随明朝将领郑芝龙, 而且施琅随后也跟随郑成功起兵反清。根据传 统正统观, 真的不忠的是郑成功。郑成功放着明朝的宗室不立, 却自立为王, 是最大的不忠。

争论的第二点基本是, 清朝算不算中国? 算的话从什么时候开始? 一种观点认 为。清朝政权慢慢接受汉族文化, 使用汉族官员, 设立汉文化学校, 恢复了科 考取士制度，满族八旗子弟均读四书五经等，这些措施使满清取得了其统治的 合法性。特别是康熙亲政，满清政权的合法性努力经历了较长的时间，从入关 到后来施琅平台, 这个过程基本上已经走完。可以说, 施琅平台时期的满清通 过自己在政治、经济、文化政策上的努力, 已经逐步获得了当时士大夫的认

同，并取得了华夏正统的地位。

\footnotetext{
13 “陈明就《施琅大将军》电视剧答《新快报》记者问”，世纪中国网 http://www.cc.org.cn/newcc/browwenzhang.php?articleid=6711, 2006 年 7 月 5 日查阅。

14 同上。

15 太俊超级论坛http://bbs.taisha.org/viewthread.php?tid=430143\&page=69\&extra=page\%3D1, 2006 年 7 月 9 日查阅。
} 
这种观点认为, 满人入关初期可以看作是野蛮民族的入侵与征服, 对他们的抵 抗是英雄之举。但是满人打下天下后, 迅速地汉化、接受中国传统文化思想, 满族已逐渐成为中华民族大家庭的一员。康熙王朝推行的政策符合中华民族的 总体利益, 维护了中华民族的利益。很明显, 持这种观点的人有个没有言明的 前提, 那就是中国是以华夏为正统的国家, 否则就不是中国。那华夏是什么 呢?

陈明虽然声称是个儒家，但他也利用后殖民主义的概念来为施琅和《施》剧辩 护。他著名的三句话是, “历史是生长的, 民族是建构的, 文化是开放的”。16 陈认为, 到施琅攻台时, 清廷已经全面确立了自己的统治, 并且通过政绩和文 化归化逐渐获得了自己的政权合法性, 已经由一个 “入侵者中国者” 转换成为 中国 “统治者”。

陈明说, “我们所处的今日中国, 是一个在世界近代史进程确立的多元一体之 “五族共和' 的政治架构。如果无视这一基本事实, 株执夷夏之辨的文化中心 主义, 将民族文化的差异可以放大凸显, 只会是仇者快而亲者痛。如果真有什 么“政治不正确”，应该是夷夏之辨中的汉族中心论”。 ${ }^{17}$

异族新王朝的正当性即 “正统” 如何才能确立? 陈明认为, 从历史事实以及处 理这一问题的法律来看有两点：第一，征服者占领的有效性；第二，其对被征 服者民间或传统价值的认同程度。这就是 “土地无常主”、“社稷无常奉”。 否则怎么解释孔子对管仲的称道? 怎么解释 “非我族类，其心必异” ? 怎么解 释《春秋》 “内其国而外诸夏, 内诸夏而外夷狄” 的 “同心圆” 式 “差序结 构” ? ${ }^{18}$

但是陈明被指责为 “伪儒”、“汉奸”、“卖国贼”。很多人的攻击直指儒 学。自去年 “郑家栋事件” 以来, 儒学似乎又遭遇了第二次 “信任危机”。如 果他投清不是汉奸行为, 文天祥、岳飞又作何评价? 怎么评价抗日战争英雄 呢? 如果有谁再来一次对中国的入侵, 中国怎么办? 陈明则反驳说, 如果施琅 统台不是爱国行为, 不是实现国家统一和领土完整之举, 这岂不是承认国家分 裂是合法的吗? 而当时清统治的中国就是中国。满人初始时是屠夫和侵略者, 后来融合为统治者。换言之, 满清入关直到完全融合为五族共和的一分子这样 一个历史事件应该理解为一个在时空坐标中逐渐发生变化的过程。19

陈明主张把施、郑两家的私怨与大陆、台湾关系区分开, 把施的私德与收复台 湾的事业区分开，把康熙作为国家的代表与满汉冲突区分开，从而肯定施在当 时为着国家统一和版图完整所做的巨大贡献。他不讳言自己是从中国当下现实 利益出发看待历史。他明确认为, 施琅不是民族败类、汉奸和卖国贼, 而是有 功于中华民族和整个中国的民族英雄。

\footnotetext{
${ }^{16}$ 陈明: 《辩护与驳诘: 就施琅问题回蒋庆》, 世纪中国网 http://www.cc.org.cn/newcc/browwenzhang.php?articleid=7154，2006 年 7 月 4 日查阅。

17 同上。

18 “陈明就《施琅大将军》电视剧再答《新快报》记者问”, 世纪中国网 http://www.cc.org.cn/newcc/browwenzhang.php?，2006 年 7 月 9 日查阅。

19 同上。
} 
另一种针锋相对的观点认为, 施琅变节降寇、助清灭明, 是汉民族之败类, 即 便有功于全国统一，也是第二位的，不能改变他协助野蛮满人奴役文明的性 质。再联系着满清入关以后一路屠城的滔天罪行, 则施之罪已不止是变节、失 德，更是民族的千古罪人，愧对作为民族精魂的岳武穆、文少保等先烈。

在这场争论中, 颇有名声的儒家蒋庆在许多人的要求下, 作了以下声明来告网 友:

来函收悉, 我未看《施》剧, 不便评论。汝等非要我表态, 依我对施琅 的有限了解，施琅是明之式臣而非英雄。就当时情势论，明是夏清是 夷，明是中国正朔所在清是外逆，故施琅降清于文化上是弃夏归夷，于 政治上是明正统之叛臣, 施琅与郑氏恩怨不能成为其降清托词。施琅大 节既亏，身名瓦裂，不管降清后有何功绩，士大夫名节已坏，余事皆不 足论。儒家不以成败论英雄, 若施琅是英雄, 其如荿山一堂师友自裁殉 国何？夫子不与以德报怨，其理正与此同。至于施琅统一台湾，实与文 不与而如其仁。虽然，又岂可因之而洗却式臣污名耶！蒋庆谨复。 ${ }^{20}$

虽然蒋庆也说 “应该从中华民族的整体利益、长远利益和中国文化的价值要求 来思考问题，不应该具有狭险的种族主义倾向，这就是《春秋》“夷夏之辨” 的根本含义”， ${ }^{21}$ 蒋庆的观点显然与陈明不同。蒋庆不赞同以古为今用的方法 来建构历史。他腰间收入假道学正统。所以从自由主义的观点来看蒋庆对陈明 的批评显然是不够的。蒋庆主张 “以王道主义超越民族主义”, 认为孔子王心 所追求的王道政治是 “任徳不任力” 的道德的政治, 而民族主义在本质上则是 “任力不任徳” 的不道德的政治。但蒋庆如何能避开民族主义的话题? 其他不 说, 夷夏之辨本身的文化民族主义倾向显然是无可置疑的。退一步讲, 如果以 公羊学所主张和蒋庆所接受的 “夷夏是以文化论（暗含与血缘、利益无涉之 意）”, 那么, 今日之天下谁是夷谁是夏恐怕还是一个需要讨论的问题。自由 主义者问，儒家学者钱穆先生访美，不是曾发出 “三代之治在美国” 的感慨 么？ 新儒家强调建立区别于西方的 “我们的” 民族国家，强调民族国家即使不 是在制度上，至少也是在文化、精神上的 “中国性”。自由主义者争辩说，新 儒家人物似乎忘了, 民族国家理念及民族主义心态, 恰是他们所要抵抗的近现 代西方的核心理念和制度，而完全有悖于儒家传统理念。 ${ }^{22}$

既然要用现代西方概念来建构民族主义，那就不能以汉人为中心了。那么 1999 年朱健国在其《不与水合作》一书中把吴三桂和孔子一样说成是 “国际主义 者” 就没错了。正是吴带清兵入关使关内关外成了中国。那么秦桧也应该 “站

\footnotetext{
20 见http://www.cc.org.cn/newcc/browwenzhang.php?articleid=7155, 来源: 儒学联合论坛 http://www.yuandao.com, 2006 年 7 月 12 日查阅。

21 汉风唐云 “新儒家与汉族主义激辩《施琅》, 自由派火中取栗? ” ,

http://www.tianya.cn/New/PublicForum/Content.asp?flag=1\&idWriter=0\&Key=0\&idArticle=229322 \&strItem $=n 001,2006$ 年 7 月 10 日查阅。

22 秋风 “儒家悖论” , 世纪中国, http://www.cc.org.cn/newcc/browwenzhang.php?articleid=7156, 2006 年 7 月 12 日查阅。
} 
起来”。如果秦桧站起来了, 岳飞是不是该趾下? 其实, 以汉人为中心的中国 民族主义在九十年代曾国藩热之争论时已经受到了挑战。 ${ }^{23}$ 因为 “满人统治的 中国应是合法的中国”，“汉人和满人同时中国人”。 ${ }^{24}$

与传统儒家的思维路径不同, 自由主义者提到现代中国的政制合法性问题。在 他们看来, 陈明对施琅的翻案, 以施琅的武力统一台湾来为现行的国家统一政 策提供历史的辩护, 不仅是愚蚟的, 更是提倡不正义。因为, 任何一个现代国 家其存在的首要基础便是其统治的合法性。而这不言自明的合法性就是民主自 由。

自由派要的是自由, 不要国家。他们对于陈明新儒家的批判, 与其说是 后者没有为作为现代民族国家的中国（以大陆政权为法权主体）给出统 一国家的正当性证明，特别是没有为通过武力统一国家行为给出正当性 证明, 不如说是后者承认了民族国家这个存在本身, 即便是获得了正当 性的国家本身。在他们看来，国家这个法权主体是没有必要存在的，甚 至自由政制国家都是不存在的, 国家只是一个外部视角下的虚假拟制, 中国的问题只有一个内部视角, 就是去国家（主义）。因此, 陈明新儒 家诉诸现代民族国家的理论变革没有出路，即便解决了民族国家的正当 性问题。按照秋风的一厢情愿, 陈明最好是重新回到传统主义, 并根据 中国的普通法宪政主义开出一个儒家现代自由主义来。对于秋风恐惧

（专制独裁）国家的苦心，我是理解的，对于他的普通法思路，我在理 论上也是支持的, 但是, 我认为他的理论是不现实的, 是片面的, 充分 表现出了公共自由派的幼稚病。

从陈明和蒋庆的观点来看, 新儒家内部在关于何谓汉奸、民族英雄的问题, 何 谓夏夷之辨、王朝正统的问题，何谓立德立功、文攻武卫的等问题上产生了分 裂。陈明古为今用的思想观点与儒家联合体中的汉族主义、文化保守主义分道 扬钽。秋风等自由派的介入则用自由民主的范畴来批判儒家文化民族主义。

在告别革命话语构建中起过重要作用的李泽厚也介入了争论。李认为, 施琅不 值得称道。他认为 “岳飞、文天祥、史可法、郑成功这些人是民族英雄, 这不 应有疑问，吴三桂、洪承畴、施琅不能是民族英雄。这里不能是非不明”。 ${ }^{26}$ 李认为, 满人入侵中国是历史的倒退, 不能以现在的概念把那时的满人称为民 族兄弟。李认为, 没有民主政治而大谈民族主义是危险的。 ${ }^{27}$ 李的观点是很令

\footnotetext{
${ }^{23}$ Yingjie Guo 'Reimagining the Chinese Nation: The Zeng Guofan Phenomenon', Modern China, vol 2 (April), 1999, pp. 142-170.

${ }^{24}$ Shanhe Xu, quoted in Guo 2001, p. 150.

25 高全喜: 《大陆新儒家吊诡与公共自由派的幼稚病一一关于施琅问题引发的几点理论思 考》，世纪中国http://www.cc.org.cn/newcc/browwenzhang.php?articleid=7157，2006 年 7 月 1 日 查阅。

26 李泽厚先生关于施琅问题的访谈 - China History Forum, online chinese http://www.chinahistoryforum.com/index.php?showtopic=11271，2006 年 7 月 13 日查阅。

27 李泽厚先生关于施琅问题的访谈 - China History Forum, online chinese http://www.chinahistoryforum.com/index.php?showtopic=11271，2006 年 7 月 13 日查阅。
} 
人费解的：反对谈民族主义的同时怎么能宣称 “岳飞、文天祥、史可法、郑成 功这些人是民族英雄” 呢?

\section{结论：中国文化民族主义困境的三个原因}

《施琅大将军》电视剧所引发的争论使我们很清楚地看出, 中国文化民族主义 复兴的困境至少有三大原因。第一个原因来自什么是中国文化的难题。首先就 有一个谁是中国人的问题。如果我们同意根据现代民族国家的定义来讨论，认 定所有在中华人民共和国境内的公民都是中国人，那么，中国文化应该包括什 么？是四书五经文本里的内容吗？是仁、义、礼、智、信、忠、孝、节吗？是 天命论吗? 是道德经吗? 如果是的话, 那么其他的所谓少数民族的文化就不是 中国文化了。佛教是不是中国文化的一部分? 穆斯林文化算不算中国文化的一 部分？算的话中国文化民族主义复兴是复兴什么呢？不算的话，怎么能把汉人 文化复兴叫成是中国文化民族主义复兴呢? 陈明和蒋庆两位所谓儒家分歧的关 键就在这里。蒋庆显然是关心汉民族文化问题的。而陈明则要关心现代民族国 家的问题。这就使他碰到了麻烦。很多网上批评陈明的言论都是从汉民族文化 为出发点的。

中国文化民族主义的困境的第二个原因与第一个是相关的，即民族国家确认性 难题。我们知道, 民族国家（nation state）是现代政治概念和政治实践。因 此, 一方面可以说在没有民族国家这一范式作为评判战争行为的标准之前, 满 人入关不能说是真正意义上的对主权国家的入侵, 所以抵抗的人不能说是真正 意义上的爱国, 投降的人也不能说是真正意义上的卖国。另一方面又可以说国 与不国不只是定义和国际法的问题，也是一个人类相处的实践问题。任何一个 居民区都有一个团体利益的问题。保卫这个居民区的团体利益的行为应该是比 出卖这个居民区的团体利益行为更值得赞赏。如果与之相反的价值得到鼓励, 任何居民区的人都不能安居乐业。况且，入侵者在入侵时实施的残暴从任何道 德原则和政治定义上来说都是应该谴责的。所以陈明会说, 满人入关之初抵抗 是英雄，但当这些入侵者成为正统的中国统治者时，为这个政权服务也是应该 的, 而且满人接受汉族文化, 以汉族文化价值来统治中国。这个正统是汉族文 化的正统，统一台湾也是为这个汉族正统服务的。

但正统并不等于正义。否则任何强者只要有能力强占住就是合法的了。实际 上, 人类历史也基本上是如此。所以中国才有强者为王败者为贼的说法。但是 作为人类, 我们总是不断地寻找和追求正义或合法的范式来规范人类行为, 否 则就跟动物没有差别了。这实际上的从林法则和理论上的正义追求是民族国家 确认性艰难的根源。

中国文化民族主义复兴困境的第三个原因从《施琅大将军》电视剧争论中自由 主义者的言论中可以看出来, 那就是, 中国文化一一应该说是汉族文化一一不 能作为评判是非和正义的标准。根据五四运动的传统, 自由主义者直截了当地 说, 汉族文化是落后的, 甚至是反人性的, 只有西方民主自由文化价值能给人 类带来幸福和繁荣。从这普世的、永恒的和超国界的标准来看, 汉族文化有什 
么好复兴的? 同样的推理，一个非民主非自由的大陆攻打一个民主自由的台湾 岛就是不正义的。以杜维明为代表的海外新儒家努力从儒家古典中寻找自由和 人性的积极因素, 但是这些努力既没有跟大陆新儒家沟通达成共同点也没能说 服中国的自由主义者。当大陆新儒家呼吁从传统价值中寻找精神支柱时，自由 主义者认为这不仅是倒退而且是可笑的。与大陆新儒家的观点相反, 自由主义 者认为, 当前中国大陆存在的社会和精神问题恰恰是没有民主和自由的结果, 不是中国人丢失了好的传统，而是中国人丢得还不够。

看来, 只有在西方的自由民主和市场经济的发展和现代化模式彻底破产（如果 有这一天的话），而且中国能为人类现代发展提供新的模式后（如果有可能的 话），中国汉族文化民族主义复兴才能摆脱困境。

\section{参考书目}

Chen, Xiaomei, 1995, 'Occidentalism- A Theory of Counter-Discourse' in Post-Mao China, New York: Oxford University Press.

Gao, Mobo, 1992, 'Democracy, What Democracy? China's Road to Modernisation', China Report (New Delhi), Vol. 28, No. 1 (Jan.-March).

Gao, Mobo, 2006, 'Chinese What Chinese: The Politics of Authenticity and Ethnic Identity' in Lee Guan Kin, ed., National Boundaries and Cultural Configurations, Centre for Chinese Language and Culture and Global Publishing Co. Pte. Ltd.

Guo, Yingjie, 2001, 'The politics of national identity' in post-Tiananmen China: cultural nationalism v. state nationalism, University of Tasmania, $\mathrm{PhD}$ thesis.

Guo, Yingjie, 2004, Cultural Nationalism in Contemporary China: The Search for National Identity under Reform, London and New York: RoutledgeCurzon.

Guo, Yingjie, 1999, 'Reimagining the Chinese Nation: The Zeng Guofan Phenomenon', Modern China, vol 2 (April).

Townsend, James, 1996, 'Chinese nationalism’ in Jon Unger, ed., Chinese Nationalism, Armonk, New York: M. E. Sharpe.

李泽厚: 《告别革命: 回望二十世纪中国》, 香港: 天的图书有限公司, 2004 年第 5 版。

李喜所：《“中华民族” 概念为梁启超最先提出》，中新网。

本文作者高墨波系澳大利亚塔斯马尼亚大学副教授, 其研究涉及中国当代的社 会和政治问题。他发表了大量的学术论文，主要著作有《高村》等。 